nucl-th/9407002

\title{
Friedel Oscillations in Relativistic Nuclear Matter
}

\author{
Joaquin Diaz Alonso \\ D.A.R.C., Observatoire de Paris-Meudon, 92190 Meudon, France \\ E. Gallego and A. Pérez \\ Departamento de Física Teórica, Universidad de Valencia 46100 Burjassot (Valencia), Spain
}

\begin{abstract}
We calculate the low-momentum N-N effective potential obtained in the OBE approximation, inside a nuclear plasma at finite temperature, as described by the relativistic $\sigma-\omega$ model. We analyze the screening effects on the attractive part of the potential in the intermediate range as density or temperature increase. In the long range the potential shows Friedel-like oscillations instead of the usual exponential damping. These oscillations arise from the sharp edge of the Fermi surface and should be encountered in any realistic model of nuclear matter.
\end{abstract}

21.65.+f,21.30.+y,21.60.Jz 
In the usual approaches to the study of nuclear matter near saturation density, the phenomenological potentials are basic ingredients for the description of the nuclear interaction in a Schrödinger-dynamical framework [1]. Potentials obtained from the exchange of single pseudoscalar, scalar, pseudovector and vector mesons with different theoretical approaches [2] lead to satisfactory quantitative predictions of the observed properties of two-nucleon systems. A simplified description of the main features of the $\mathrm{N}-\mathrm{N}$ interaction can be obtained from the exchange of scalar and vector mesons only [3].

For higher densities (owing to the Pauli principle) relativistic effects become essential, not only in the description of the interaction itself, but also for the analysis of the particle dynamics. In this case, the Lagrangian approach becomes the natural framework for the study of the nuclear plasma [4]. The solution of appropriate Lagrangian models in the relativistic Hartree approximation (RHA) provides a satisfactory picture of the thermodynamical behaviour of relativistic nuclear matter at finite temperature [5]. Moreover, the relativistic meson propagators in vacuum obtained from this approach allow for the calculation of the $\mathrm{N}-\mathrm{N}$ interaction potentials [6].

Inside nuclear matter, the polarization effects introduce important changes in the form of the relativistic meson propagators. Consequently, the $\mathrm{N}-\mathrm{N}$ interaction potentials inside the plasma are also strongly modified by the screening. Their behaviour as a function of the thermodynamical state provides a very explanatory visualization of these effects and suggest the existence of new collective phenomena.

In this letter we report some results of a study of the $\mathrm{N}-\mathrm{N}$ interaction potentials obtained in the one-boson exchange model (OBE) inside symmetric nuclear matter at finite temperature, and analyze some phenomena related to the screening.

The Lagrangian model used for this calculation describes the nuclear interaction in terms of scalar- $\sigma$ and vector- $\omega$ mesons exchanges [3]. Although such a simplified model is not able to account for the whole richness of actual nuclear matter, it gives (when solved in RHA [7]) an acceptable description for its thermodynamical behaviour. Moreover, as mentioned above, the involved mesons reproduce the main qualitative features of the nuclear interac- 
tion in vacuum. In fact the fictitious $\sigma$-meson, which provides the attractive part of the potential at intermediate range, is introduced as a simple parameterization of the correlated $2 \pi$-exchange contribution to the $\mathrm{N}-\mathrm{N}$ interaction. It has not been proven that such a parameterization is also possible inside the nuclear medium. Nevertheless, the behaviour of the screened potential with the thermodynamical state found here is qualitatively similar, in the intermediate range, to the one found in a $2 \pi$-exchange calculation 8 . Moreover, as we shall see, the medium effects on the long-range behaviour of this potential are mainly dominated by the singularities of the matter polarization contributions, and are rather independent of the details of the basic interaction. They should be present in more realistic analysis which take into account all the relevant meson exchanges.

When solving the model in the RHA, the values of the constants in the Lagrangian are fixed as follows: The meson and fermion masses are fixed to their "physical" values $\mu_{\sigma}=550 \mathrm{MeV}, \mu_{\omega}=783 \mathrm{MeV}$ and $m=939 \mathrm{MeV}$. For the coupling constants, we choose the values which lead to a satisfactory fit of the saturation properties in the RHA: $g_{\sigma}^{2}=183.3\left(\mu_{\sigma} / m\right)^{2}$ and $g_{\omega}^{2}=114.7\left(\mu_{\omega} / m\right)^{2}$. From these values, saturation is attained at a Fermi momentum of the nucleon $P_{f 0}=1.42 \mathrm{fm}^{-1}$, with a binding energy $E_{b}=-15.46 \mathrm{MeV}$.

In going beyond RHA, the analysis of the small perturbations of the fermion distribution and meson fields around the Hartree equilibrium gives the expressions for the meson propagation equations inside the plasma, which can can be written in a matrix form as $D(k) \cdot \Sigma_{1}(k)=0$, where $\Sigma_{1}$ is the column matrix of the components of the scalar and vector perturbing fields, and $D(k)$ is a $5 \times 5$-matrix containing the scalar $\left(\Pi_{\sigma}\right)$, mixing $\left(\Pi_{\sigma \omega}^{\mu}\right)$ and vector $\left(\Pi_{\omega}^{\mu \nu}\right)$ polarization tensors, which are functions of the thermodynamical state and include the renormalized vacuum contributions [9]. At $\mathrm{T}=0$ they reduce to the one-loop meson polarizations [10]. The propagator matrix for the mixed scalar-vector field inside matter is given by 9, 11$] G(k)=-D(k)^{-1}$. At zero density and temperature, the meson fields decouple from each other, and the components of this matrix reduce to the one-loop scalar and vector propagators in vacuum.

The first step in the calculation of the screened two-nucleon potential is the derivation 
of the relativistic one-boson $(\sigma+\omega)$ exchange amplitude diagrams from the propagator. Now, the difference with the calculation in vacuum [6] is that the meson propagators are dressed by the medium, and mixing between both meson fields appears [9]. Therefore, the usual Feynman rules are slightly modified in the present case: We must introduce a $i \Gamma_{a}$ factor at each vertex ( $\Gamma_{a}$ is the coupling matrix defined as $\Gamma_{\mu}=\gamma_{\mu} g_{\omega}$ for a $=\mu$ from 0 to 3 , and $\left.\Gamma_{4}=g_{\sigma}\right)$, and a dressed boson propagator matrix $i G(k)$ for each internal boson line. Moreover, in the present calculation we are interested in the structure of the propagator matrix on the $k^{0}=0$ axis only, where there are two poles associated to the "tachyonic" branches, coming from the vacuum polarization terms [9]. Such poles are spurious because they arise at large values of q, where the point-particle approach fails, and the nucleon structure should be taken into account. This is done through the introduction of phenomenological monopolar form factors:

$$
f_{a}(k)=\left(\Lambda_{a}^{2}-\mu_{a}^{2}\right) /\left(\Lambda_{a}^{2}-k^{2}\right)
$$

$(a=\sigma, \omega)$

at each vertex of the loop and boson-exchange diagrams. This amounts to multiplying each squared coupling constant by the corresponding form factor. With this prescription the spurious "tachyonic" branches (and the associated poles in the propagator matrix) disappear [9].

Under these conditions, the relativistic OBE amplitude takes the form

$$
\begin{gathered}
A=\quad\left\{\left[\chi_{1^{\prime}}^{\dagger} \bar{u}\left({\overrightarrow{p^{\prime}}}_{1}, s_{1}^{\prime}\right)\right](i \Gamma)_{m}\left[u\left(\vec{p}_{1}, s_{1}\right) \chi_{1}\right]\right\} \\
\cdot(i G(k))^{m n}\left\{\left[\chi_{2^{\prime}}^{\dagger} \bar{u}\left(\overrightarrow{p^{\prime}}, 2, s_{2}^{\prime}\right)\right](i \Gamma)_{n}\left[u\left(\vec{p}_{2}, s_{2}\right) \chi_{2}\right]\right\}
\end{gathered}
$$

where $p_{1}$ and $p_{2}$ are the four-momenta for the incoming quasi-nucleon states and $p_{1}^{\prime}$ and $p_{2}^{\prime}$ correspond to the outgoing quasi-nucleon states, whereas $k=p_{1}-p_{1}^{\prime}=p_{2}^{\prime}-p_{2}$ is the transferred four-momentum. The interacting quasi-nucleons have effective mass M (as given by the RHA approximation) and spins $\vec{S}_{1}=\frac{1}{2} \vec{\sigma}_{1}$ and $\vec{S}_{2}=\frac{1}{2} \vec{\sigma}_{2}$. The indices $\mathrm{m}$ and $\mathrm{n}$ run from 0 to 4 . Finally $u(\vec{p}, s)$ and $\chi$ are the Dirac cuadri-spinor and the isospin wave function, respectively. 
The potential (OBEP) is obtained by eliminating the wave functions of the initial and final states in the amplitude Eq. (2) taken in the center-of-mass system. From this expression we obtain the non-relativistic potential by performing an expansion in the nucleon momenta and keeping only the second-order terms. We have corrected the OBEP according to the Blankenblecker-Sugar prescription [12], which includes the requirement of "minimal relativity" [6] as defined by $V\left(q^{\prime}, q\right)=\left(M / E_{q^{\prime}}\right)^{1 / 2} V_{O B E P}\left(q^{\prime}, q\right)\left(M / E_{q}\right)^{1 / 2}$ where $q$ and $q^{\prime}$ are the CMS initial and final momenta of the nucleons, respectively, $E_{q}=\sqrt{M^{2}+q^{2}}$, and $E_{q^{\prime}}=\sqrt{M^{2}+q^{\prime 2}}$. Moreover, the above prescription implies taking the static $\left(k^{0}=0\right)$ limit. After Fourier transformation one obtains the potential in coordinate space:

$$
V(\vec{r})=V_{c}(r)-\frac{1}{2}\left(\nabla^{2} V_{2}(\vec{r})+V_{2}(\vec{r}) \nabla^{2}\right)+V_{L S}(r) \vec{L} \cdot \vec{S}+V_{S S}(r) \vec{\sigma}_{1} \cdot \vec{\sigma}_{2}+V_{T}(r) S_{12}
$$

where $\vec{L}=\vec{r} \wedge \vec{p}$ is the orbital kinetic momentum, $\vec{S}=(1 / 2)\left(\vec{\sigma}_{1}+\vec{\sigma}_{2}\right)$ is the total spin operator and $S_{12}=\frac{3}{r^{2}}\left(\vec{\sigma}_{1} \cdot \vec{x}\right)\left(\vec{\sigma}_{2} \cdot \vec{x}\right)-\left(\vec{\sigma}_{1} \cdot \vec{\sigma}_{2}\right)$ is the tensor operator.

The second term in Eq.(3) is a non-local component which gives a small contribution to the potential. We shall omit here the study of this component. The other terms (central, spin-spin, spin-orbit and tensor components) are now functions of the interparticle distance, as well as of the thermodynamical state of the plasma (density and temperature). We must emphasize that the non-relativistic limit concerns the dynamics of the two interacting nucleons and the neglecting of retardation effects. However, the description of the thermodynamical state of the plasma in the RHA remains fully relativistic. Also, no low-q approximation has been done for the meson propagators.

In the analysis of the potential, the values of the coupling constants and cut-off parameters in the form factors have been fixed in order to fit the deuteron and low-energy phenomenology data [6]:

$$
g_{\sigma}^{2}=8.7171 \quad g_{\omega}^{2}=25 \quad \Lambda_{\sigma}=2.0 \mathrm{GeV} \quad \Lambda_{\omega}=1.4 \mathrm{GeV}
$$

However, in calculating the underlying thermodynamical state, the coupling constants have been fixed to the above mentioned values which fit saturation in RHA. 
Figure (1) is a picture of the central component of the potential at $T=0$, at saturation density (solid line) and 2.4 times these density (dashed-dotted line), for symmetric nuclear matter ( $P_{f}$ is the Fermi momentum of the nucleons). The same component, calculated at zero density, with $\left(V_{p o l}\right)$ and without $\left(V_{v a c}\right)$ vacuum polarization contributions has also been plotted. The very short-range region $(r<0.6 \mathrm{fm})$ must be discarded because the potential behaviour is dominated there by large-q values, and the non-relativistic (low-q) limit breaks down [11]. Beyond this distance, a hard-core and a potential well appear both in vacuum and at finite density. At zero density, the vacuum polarization effects enhance the slope of the hard-core and increase the depth of the potential well by an important amount. The slope of the repulsive-vector Yukawa component is strongly raised by the vacuum polarization, whereas the attractive-scalar component remains nearly unaffected by these effects. This explains the observed behaviour in vacuum. The matter polarization effects increase the range of the vector component, and therefore the depth of the well is reduced as density grows. It disappears shortly above the saturation density [11. Similar results are obtained if the attractive $\sigma$ contribution to the $\mathrm{N}-\mathrm{N}$ potential is replaced by the $2 \pi$-exchange [\$] .

At larger distances, the screening effects of the medium introduce important qualitative new features. Whereas in vacuum the potential shows an exponential damping with the distance, an oscillatory behaviour appears at finite density, whose amplitude is damped as an integer power of the distance. These are Friedel oscillations, similar to those encountered in many low-temperature Fermi systems [13]. Mathematically, these oscillations arise from the fact that the matter polarization contributions to the screened meson propagators show singularities in their derivatives at $q=2 P_{f}$ (Kohn singularities [14]). After Fourier transformation, such singularities introduce oscillations in the r-space potential. More detailed analytical calculations [15], [19] show that, in this region, the potential can be decomposed into $\sigma$ and $\omega$ Yukawa-like components (which dominate at shorter distances), and this Friedel-like component, which determines the large-r features and is long-ranged and oscillatory. From a physical point of view, the Kohn singularity is associated to the sharp character of the Fermi surface at $T=0$ and is rather independent on the details of the 
interaction. Consequently, the long-range oscillations of the screened interparticle potential must be an universal feature of interacting degenerate Fermi systems. Indeed, this has been found for degenerate non-relativistic [16] and relativistic [17] electromagnetic plasmas, for a QCD plasma [18], and for the screened one-pion exchange potential in a relativistic nuclear plasma [15].

In the calculation of Ref. [8], where the $2 \pi$-exchange is explicitly considered, no Friedel oscillations have been found. In fact, this paper was concerned with the intermediate range of the screened N-N potential, and the complete effects of the matter polarizations were not included in the meson propagators. Such effects go beyond the effective meson mass variation with density, considered by the authors. Therefore, it is not surprising that they do not obtain oscillations in the long range.

As a test of the small sensitivity of the Friedel behaviour to the details of the interaction, we have plotted in Fig.(1) the central component of the potential at saturation density and $T=0$ (dotted line), now using for the potential the values of the coupling constants which fit saturation in the RHA, which are very different from the ones used in the previous calculation for the same thermodynamical state. We observe important differences in the intermediate region in both cases but, in the long range, the oscillations of the potential are only slightly affected by the changes in the intensity of the couplings.

In Fig. (2) the spin-spin component of the potential is plotted at $T=0$, in vacuum $\left(P_{f}=0\right)$, at saturation density $\left(P_{f} / m=0.3\right)$, and 2.4 times saturation density $\left(P_{f} / m=\right.$ 0.4). A glance to this drawing shows also a Friedel-like oscillatory behaviour whose amplitude increases with density. (The spin-orbit and tensor components of the potential show also a similar behaviour: in all cases the amplitude of the oscillations increase as density grows [11). At saturation density, the most important effect in the long-range appears on the central potential component, where the amplitude of the first oscillation reaches $2 \mathrm{MeV}$ for a distance of around $2 \mathrm{fm}$. Nevertheless, for higher densities the oscillations of the spin-spin component reach comparable maxima. Indeed, for $P_{f} / m=0.4$ the amplitude of the first oscillation in the central component is $\sim 8 \mathrm{MeV}$ at $r_{\max } \approx 1.5 \mathrm{fm}$ (see Fig.(1)), 
whereas the spin-spin component reaches $10 \mathrm{MeV}$ at the same distance (see Fig.(2)). The position of the first maximum of the oscillations, $r_{\max }$, is related to the Fermi momentum through [15] $P p_{f} \cdot r_{\max } \approx \pi$. The ratio between the position of the first maximum of the oscillation and the mean interparticle distance $d=\left(3 \pi^{2} / 2 P_{f}^{3}\right)^{1 / 3}$ is nearly constant with density $\left(r_{\max } / d \approx 1.28\right)$.

At finite temperature, the slope of the hard core is slightly reduced, as showed in Fig.(3), where the central component has been plotted at saturation density and various temperatures. In the intermediate range, the effects of temperature on the central potential reduce the depth of the well. The well disappears for temperatures beyond $40 \mathrm{MeV}$. This can be interpreted in terms of the modifications introduced on the Yukawa-like components by the temperature in this region. As temperature increases, the ranges and intensities of these Yukawa components are modified in such a way that in the balance, the vector-repulsive part becomes dominant and the well disappears. (see Refs. [15] and [19] for a more detailed analytical study).

Concerning the long-range behaviour of the potential, as temperature increases the Fermi distribution function becomes smooth and the Kohn singularity (and the associated oscillations) disappears in all the components of the potential. This is indeed observed in Fig. (3). The amplitudes of the oscillations decrease with temperature. As can be checked analytically [15,19] this amplitude at a fixed distance and constant density is exponentially damped with temperature. The oscillations in all the components disappear for temperatures between $40-80 \mathrm{MeV}$. Beyond this, the potential becomes exponentially damped with the distance.

\section{ACKNOWLEDGMENTS}

This work has been supported in part by the spanish CICYT, Grant AEN 93-0234 and DGICYT Grant PB91-0648. 


\section{REFERENCES}

[1] A.L. Fetter, J.D. Walecka : "Quantum Theory of Many-Particle Systems". Mc-GrawHill (1971).

[2] M. Lacombe, B. Loiseau, J.M. Richard, R. Vinh Mau, J. Cote, P. Pires, R. de Tourreil : Phys. Rev. C21, (1980), 861.

R. Machleidt, K. Holinde, C. Elster : Phys. Rep. 149, (1987), 1.

[3] B.D. Serot, J.D. Walecka: "The Relativistic Nuclear Many-Body Problem." Adv. Nucl. Phys. Vol.16, J.W. Negele and E. Vogt Ed. Plenum Press, New York (1986).

J.D. Walecka : Ann. Phys. 83, (1974), 491.

[4] B.D. Serot : Rep. Prog. Phys. 55, (1992), 1855.

[5] J. Diaz Alonso: Phys. Rev. D31, (1985), 1315

[6] R. Machleidt: "The Meson Theory of Nuclear Forces and Nuclear Structure." Adv. in Nucl. Phys. Vol.19, J.W. Negele and E. Vogt Ed. Plenum Press, New York (1989)

[7] S.A. Chin : Ann. of Phys. 108, (1977), 301.

[8] J.W. Durso, H-C. Kim, J. Wambach: Phys. Lett. B 298, (1993), 267.

[9] J. Diaz Alonso, A. Perez Canyellas: Nucl. Phys. A 526, (1991), 623

[10] T. Matsui, B.D. Serot: Ann. of Phys. 144, N1, (1982), 107

[11] E. Gallego, J. Diaz Alonso, A. Perez Canyellas: "Screening Effects on the N-N Potential in Relativistic Nuclear Matter." In preparation.

[12] K. Erkelenz: Phys. Rep. 13 C, (1974), 191.

Ch. W. Wong: "Topics in Nuclear Physics I", Lecture Notes in Physics, 144, SpringerVerlag (1981)

[13] J. Friedel: Phyl. Mag. 43, (1952), 153.; 
Nuovo Cimento, 7, (1958), Suppl.2, 287.

[14] W. Kohn: Phys. Rev. Lett. 2, (1959), 393.

J.S. Langer, S.H. Vosko: J. Phys. Chem. Solids, 12, (1960), 196.

[15] J. Diaz Alonso, A. Perez Canyellas, H. Sivak: Nucl. Phys. A205, (1989), 695

[16] M.E. Rensik: Phys. Rev. 174, (1968), 744.

N.J. Horing: Phys. Rev. 186, (1969), 434

[17] H. Sivak: Physica, A129, (1985), 408.

[18] J. Kapusta, T. Toimela : Phys. Rev. D37, (1988), 3731.

[19] J. Diaz Alonso, E. Gallego, A. Perez Canyellas, H.Sivak: "Linear Response and Friedel Oscillations of Meson Fields in Relativistic Nuclear Matter." To be published. 


\section{FIGURES}

FIG. 1. Central component of the potential at $T=0$ at zero density with $\left(V_{\text {pol }}\right)$ and without $\left(V_{\text {vac }}\right)$ vacuum polarization contributions at saturation density $\left(P_{f} / m=0.3\right)$ and 2.4 times saturation density $\left(P_{f} / m=0.4\right)$. The dotted line (with $P_{f} / m=0.3$ ) corresponds to the central component of the potential at saturation density obtained from different values of the model parameters.

FIG. 2. Spin-spin component of the potential at $T=0$ for the same values of the Fermi momentum as in Fig.1.

FIG. 3. Central component of the potential at saturation density for different temperatures. 


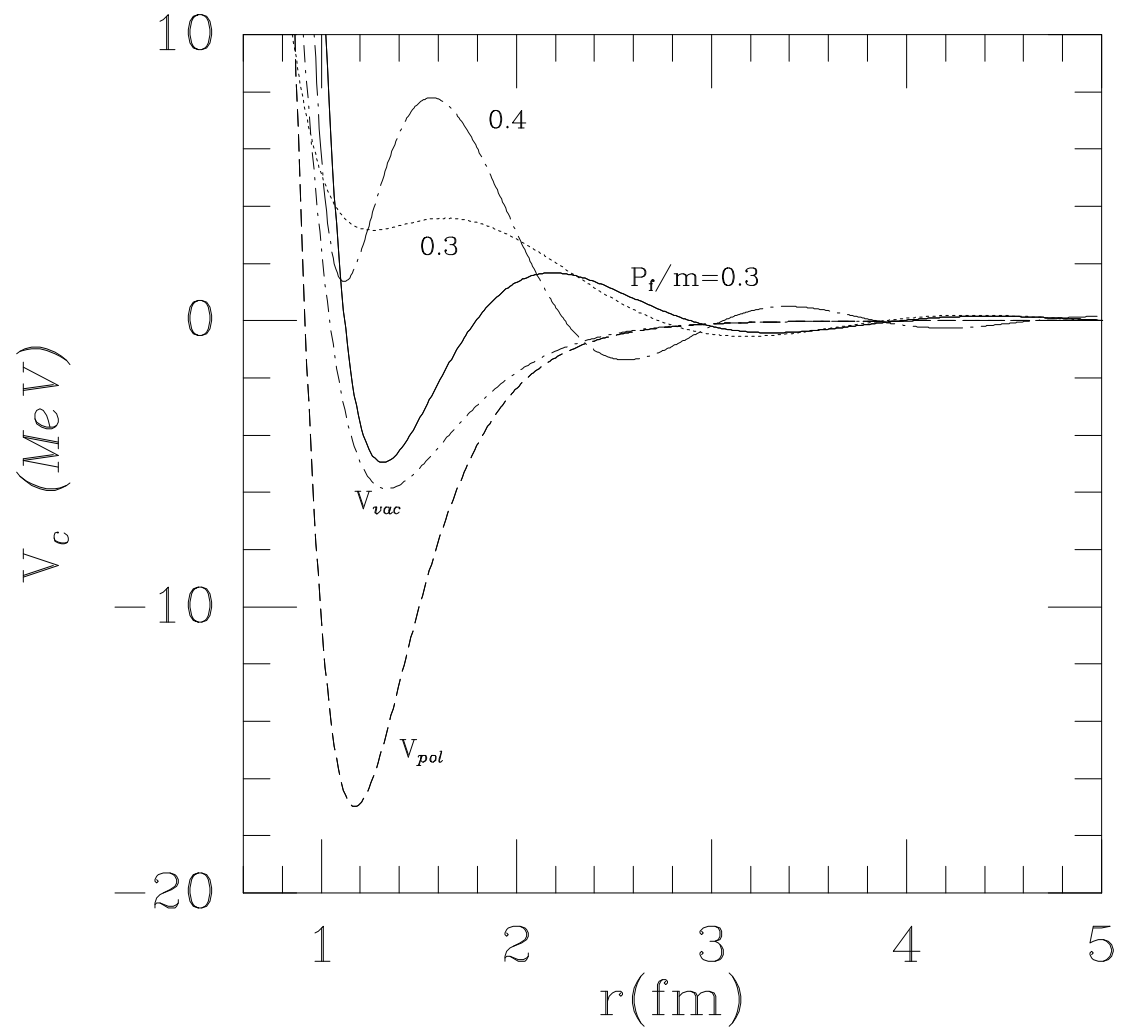

FIG. 1 
This figure "fig1-1.png" is available in "png" format from: http://arxiv.org/ps/nucl-th/9407002v1 
This figure "fig2-1.png" is available in "png" format from: http://arxiv.org/ps/nucl-th/9407002v1 
This figure "fig3-1.png" is available in "png" format from: http://arxiv.org/ps/nucl-th/9407002v1 


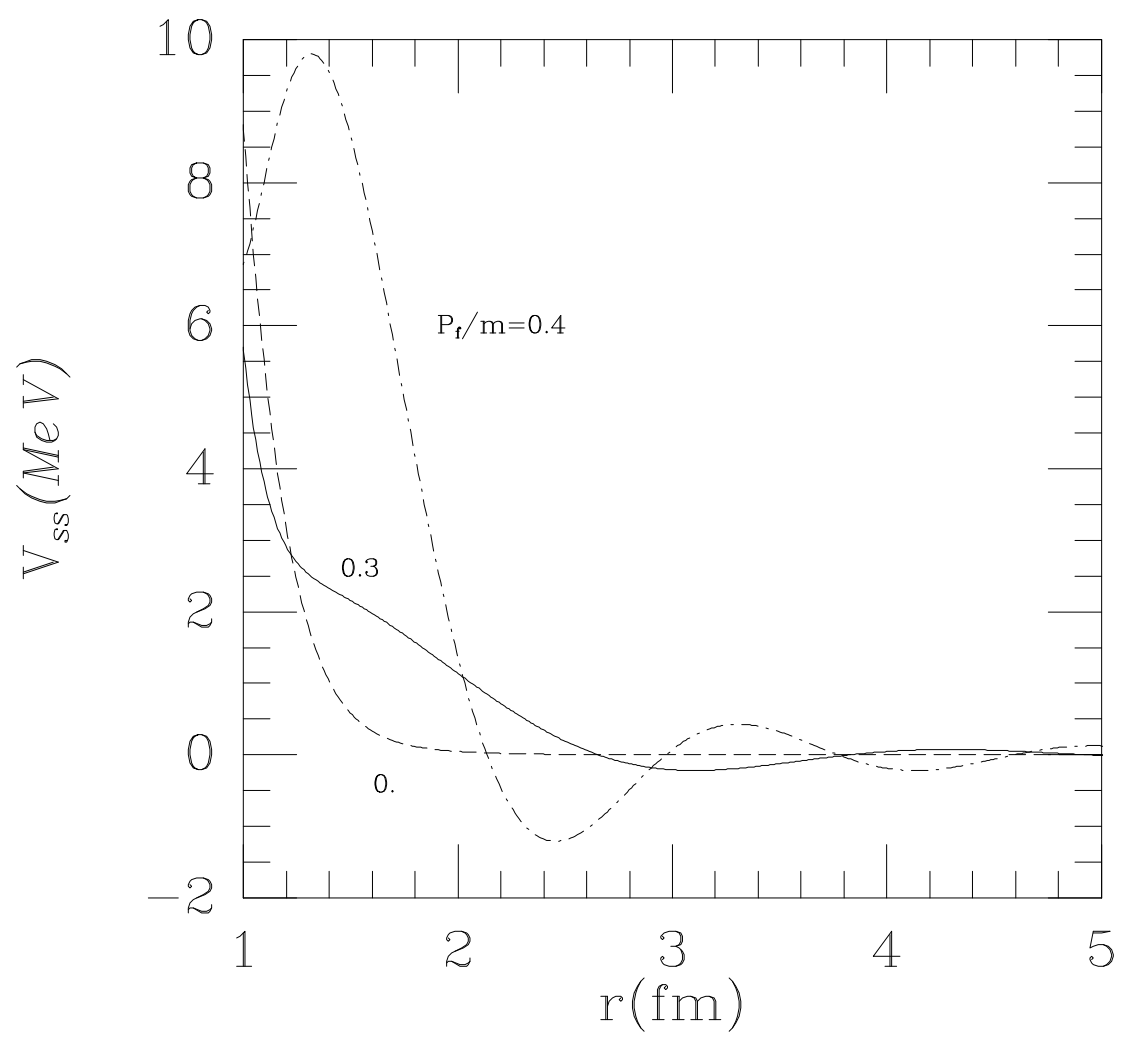

FIG. 2 


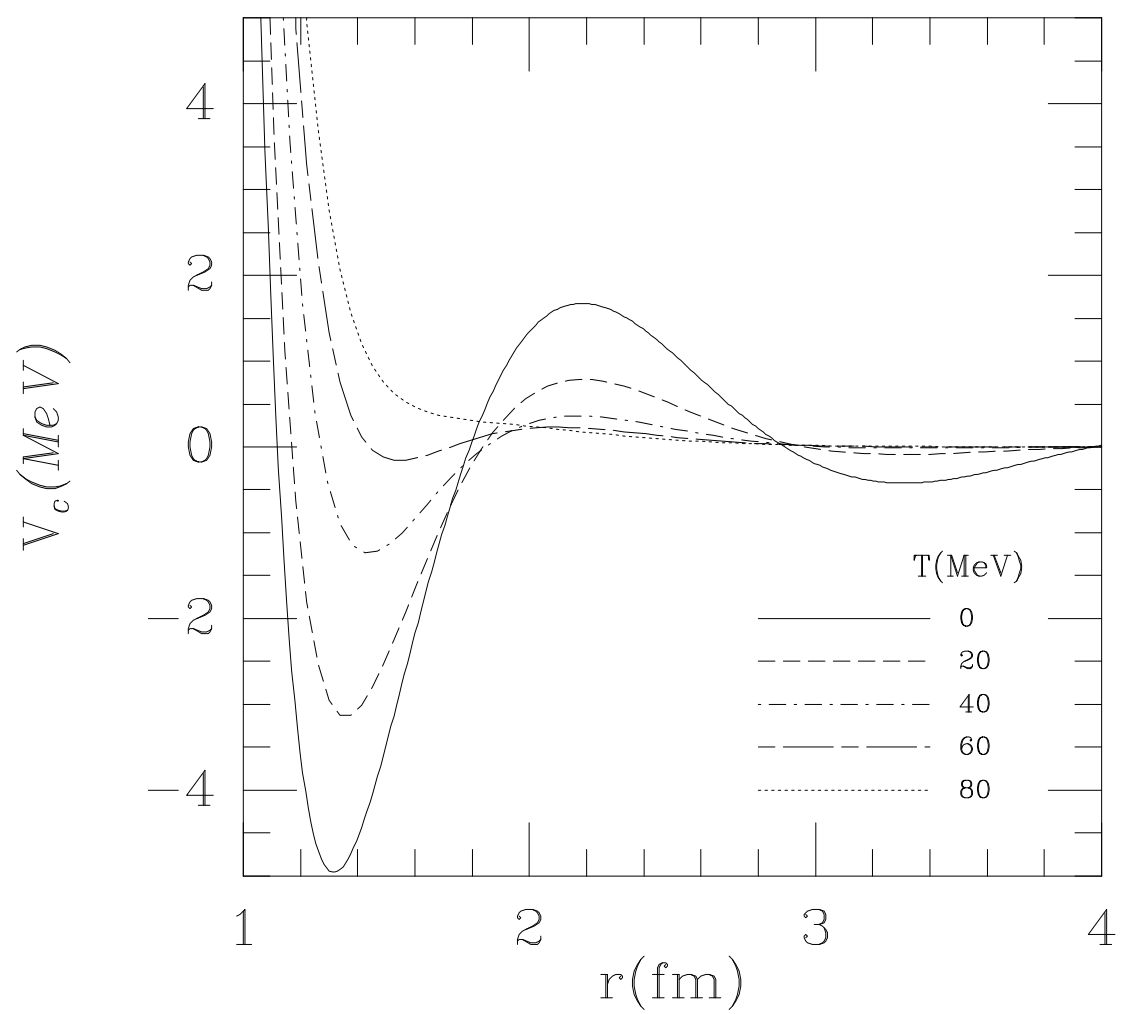

FIG. 3 